\title{
Risk-sensitive Preview Tracking Control of Stochastic Systems
}

\author{
Yuichi Sawada \\ Department of Mechanical and System Engineering, \\ Kyoto Institute of Technology \\ Matsugasaki Sakyo, Kyoto 606-8585, Japan
}

E-mail: sawada@kit.ac.jp

\begin{abstract}
This paper presents a problem of preview tracking control for discrete-time stochastic linear systems via risk-sensitive stochastic optimal control theory. The risk-sensitive preview tracking controller is designed for an augmented system consisting of a tracking error system and a command generator which makes preview information of the reference signal. In the simulation studies, it has been shown that the derived controller has successfully achieved reducing the tracking error and increasing the robustness against strong random disturbance.
\end{abstract}

\section{Introduction}

Physical systems in many fields require to be controlled so that the output tracks the reference signal. Especially, servomotors based on the tracking control technique used for mechanical systems are significant technology. The objective of such controllers is to minimize the tracking error and to meet other physical specifications of controlled objects.

It is well known that the tracking control with preview action reduces its tracking error. There are many researches of preview tracking control problems. LQ optimal controls based preview tracking control have been investigated by Tomizuka [1, 2], Katayama et al.[3] and Kojima et al. [4] and Fujisaki et al. [5]. $H^{\infty}$ control with preview action has been studied to introduce robustness $[6,7,8]$. A preview tracking control for a stochastic system has been considered by Rho and Park [9]. On the other hand, tracking control problems using risk-sensitive control were investigated by Collings et al. [10]. However the risk-sensitive tracking control with preview action has not been studied.

Jacobson has started the studies of the optimal control problem for discrete-time linear systems with exponential criteria [11]. Speyer et al. [12] and Bensoussan et al. $[13,14]$ have extended the theory to the system with observation systems. Whittle has found the relation between the $\mathrm{H}$ controls and the risk-sensitive control theories [15]. After that, many researchers investigated the risksensitive control for nonlinear systems [16], satellite systems $[17,18]$.

In this paper a problem of preview tracking control for discrete-time linear stochastic systems via the risk-sensitive stochastic optimal control theory is investigated. The risk-sensitive preview tracking controller is designed for an augmented system consisting of a tracking error system expressed by incremental states and a command generator which makes preview information of the reference signal. The controller is solved via the risksensitive control theory for discrete-time systems. Finally, several numerical results are presented.

\section{System Model}

Consider the following discrete-time stochastic linear systems:

$$
x(k+1)=A x(k)+B u(k)+G w(k),
$$

where $x(k) \in \mathbb{R}^{n}$ and $u(k) \in \mathbb{R}^{m}$ denote the state and control vectors, respectively. $w(k) \in \mathbb{R}^{q}$ represents the random disturbance vector modeled by the white Gaussian noise with $\mathscr{E}\{w(k)\}=0$; $\mathscr{E}\left\{w(k) w^{\prime}(j)\right\}=W \delta_{k j}$, where $\delta_{k j}$ denotes the Kronecker delta and the prime represents the matrix transpose. The system matrices are $A \in \mathbb{R}^{n \times n}$; $B \in \mathbb{R}^{n \times m}$ and $G \in \mathbb{R}^{n \times q}$. 
The output is supposed to be given by

$$
y(k)=C x(k),
$$

where $y(k) \in \mathbb{R}^{p}$ denotes the output vector and $C \in \mathbb{R}^{p \times n}$. The reference signal is defined by $r(k) \in \mathbb{R}^{p}$ which is given by a step type function, i.e.,

$$
r(k)=\bar{r} u_{s}(k-d)
$$

where $u_{s}(k)$ denotes the unit step function; $\bar{r}$ is a constant; $d$ the nonnegative integer. In this paper $h$ future steps of the reference signal are available to use as well as the present and past information.

The objective is to design a control law $\{u(k)\}$ such that the output vector $y(k)$ tracks the reference signal $r(k)$ without steady-state error, i.e., $\lim _{k \rightarrow \infty} e(k)=0$, where

$$
e(k)=r(k)-y(k)
$$

\section{Risk-sensitive Tracking System with Preview Action}

In order to construct the risk-sensitive preview tracking system, it is required to introduce an integrator to reduce the steady-state error. Now let us define the incremental state, control and disturbance vectors, i.e., $\tilde{x}(k):=x(k)-x(k-1)$, $\tilde{u}(k):=u(k)-u(k-1)$ and $\tilde{w}(k):=w(k)-w(k-1)$, where $\mathscr{E}\{\tilde{w}(k)\}=0 ; \mathscr{E}\left\{\tilde{w}(k) \tilde{w}^{\prime}(j)\right\}=2 W \delta_{k j}-$ $2 W \delta_{|k-j|, 1}$. The tracking error given by (4) can also be expressed by the following incremental form:

$$
\tilde{e}(k):=e(k)-e(k-1) .
$$

Recalling the relations (2) and (4), the incremental tracking error can be described by

$$
\tilde{e}(k+1)=\tilde{r}(k+1)-C \tilde{x}(k+1),
$$

where $\tilde{r}(k+1):=r(k+1)-r(k)$.

The incremental state $\tilde{x}(k)$ is a sequence governed by the following system:

$$
\tilde{x}(k+1)=A \tilde{x}(k)+B \tilde{u}(k)+G \tilde{w}(k) .
$$

Substituting (7) into (6), a system equation with respect to $e(k)$ is obtained as

$$
\begin{aligned}
e(k+1)= & e(k)-C A \tilde{x}(k)-C B \tilde{u}(k) \\
& +\tilde{r}(k+1)-C G \tilde{w}(k) .
\end{aligned}
$$

Let us define an augmented state vector $z(k):=$ $\left[e^{\prime}(k), \tilde{x}^{\prime}(k)\right]^{\prime}$, the system equation with respect to $z(k)$ can be expressed by

$$
\begin{aligned}
z(k+1)= & A_{z} z(k)+B_{z} \tilde{u}(k) \\
& +D_{z} \tilde{r}(k+1)+G_{z} \tilde{w}(k),
\end{aligned}
$$

where

$$
\begin{aligned}
& A_{z}=\left[\begin{array}{cc}
I & -C A \\
0 & A
\end{array}\right], \quad B_{z}=\left[\begin{array}{c}
-C B \\
B
\end{array}\right] \\
& D_{z}=\left[\begin{array}{l}
I \\
0
\end{array}\right], \quad G_{z}=\left[\begin{array}{c}
-C G \\
G
\end{array}\right] .
\end{aligned}
$$

The objective of this study is to track the reference signal and to reduce the tracking error and the effect of random disturbance using the risksensitive control with preview action. These requirements are encapsulated by the following infinite risk-sensitive cost functional [11]:

$$
J(\tilde{u})=\lim _{T \rightarrow \infty} \frac{1}{T \theta} \ln \mathscr{E}\left\{\exp \theta \Psi_{0, T}(z, \tilde{u})\right\},
$$

where

$$
\Psi_{j, k}(z, \tilde{u}):=\sum_{\ell=j}^{k} \frac{1}{2}\left\{z^{\prime}(\ell) Q z(\ell)+\tilde{u}^{\prime}(\ell) R \tilde{u}(\ell)\right\}
$$

and $\theta$ denotes the scalar valued risk-sensitive parameter; $Q$ and $R$ are the non-negative and positive definite symmetric matrices, respectively. For $\theta>0$ the controller works concerned to control worst case of the sample runs (risk-averse control). If $\theta=0$ then such control problems are termed risk-neutral control problems as opposed to risksensitive control problems. These controllers are equivalent to LQG controllers.

The discrete-time incremental state system can be augmented by a command generator system which models the preview part of the system:

$$
\tilde{x}_{r}(k+1)=A_{r} \tilde{x}_{r}(k),
$$

where $x_{r}(k):=\left[r^{\prime}(k+1), \cdots, r^{\prime}(k+h)\right]^{\prime} ; \tilde{x}_{r}(k):=$ $x_{r}(k)-x_{r}(k-1)$ and

$$
A_{r}:=\left[\begin{array}{cccc}
0 & I & \cdots & 0 \\
\vdots & \ddots & \ddots & \vdots \\
0 & & 0 & I \\
0 & \cdots & & 0
\end{array}\right] \in \mathbb{R}^{p h \times p h} .
$$


The incremental state of the command generator $\tilde{x}_{r}(k)$ is composed of sampled values of the reference signal over the preview horizon of length $h$. The matrix $A_{r}$ implements a shift register operation.

Now define new augmented state vector $\zeta(k):=$ $\left[z^{\prime}(k), \tilde{x}_{r}^{\prime}(k)\right]^{\prime}$. Using $\zeta(k)$, we have the following system:

$$
\zeta(k+1)=A_{c} \zeta(k)+B_{c} \tilde{u}(k)+G_{c} \tilde{w}(k),
$$

where

$$
\begin{aligned}
& A_{c}:=\left[\begin{array}{cc}
A_{z} & G_{r} \\
0 & A_{r}
\end{array}\right], \quad B_{c}:=\left[\begin{array}{c}
B_{z} \\
0
\end{array}\right] \\
& G_{c}:=\left[\begin{array}{c}
G_{z} \\
0
\end{array}\right], \quad G_{r}:=\left[\begin{array}{llll}
D_{z} & 0 & \cdots & 0
\end{array}\right] .
\end{aligned}
$$

The cost functional given by (10) with (11) can be rewritten in terms of the augmented system as:

$$
\begin{aligned}
J(\tilde{u}) & =\lim _{T \rightarrow \infty} \frac{1}{T \theta} \ln \mathscr{E}\left\{\exp \theta \Psi_{0, T}(\zeta, \tilde{u})\right\} \\
\Psi_{j, k}(\zeta, \tilde{u}) & =\frac{1}{2} \sum_{\ell=j}^{k}\left\{\zeta^{\prime}(\ell) Q_{c} \zeta(\ell)+\tilde{u}^{\prime}(\ell) R \tilde{u}(\ell)\right\},
\end{aligned}
$$

where $Q_{c}:=\operatorname{Block} \operatorname{diag}\{Q, 0\}$.

Via the risk-sensitive control theory, the controller, which minimizes the cost functional described by (14) and (15), is given by

$$
\begin{aligned}
\tilde{u}^{o}(k) & =-\left(R+B_{c}^{\prime} \hat{\Pi} B_{c}\right)^{-1} B_{c}^{\prime} \hat{\Pi} A_{c} \zeta(k) \\
& \equiv-\left[K_{e}, K_{x}, K_{r}\right] \zeta(k),
\end{aligned}
$$

where $K_{e} \in \mathbb{R}^{m \times p}, K_{x} \in \mathbb{R}^{m \times n}$ and $K_{r} \in \mathbb{R}^{m \times p h)}$ are the gain matrices and $\hat{\Pi}$ which is the solution of the algebraic Riccati equation given by

$$
\begin{aligned}
\hat{\Pi}= & \Pi+\theta \Pi G_{c}\left(2 W-\theta G_{c}^{\prime} \Pi G_{c}\right)^{-1} G_{c}^{\prime} \Pi \\
\Pi= & Q_{c}+A_{c}^{\prime} \hat{\Pi} A_{c} \\
& \quad-A_{c}^{\prime} \hat{\Pi} B_{c}\left(R+B_{c}^{\prime} \hat{\Pi} B_{c}\right)^{-1} B_{c}^{\prime} \hat{\Pi} A_{c} .
\end{aligned}
$$

Recalling the definition of the incremental state and control input, the optimal control given by (17) can be rewritten into the following form:

$$
\begin{aligned}
u^{o}(k)= & -K_{e} \sum_{j=1}^{k} e(j)-K_{r} x_{r}(k)-K_{x} x(k) \\
& +K_{r} x_{r}(0)+K_{x} x(0)+u^{o}(0) .
\end{aligned}
$$

\section{Numerical Results}

Here several simulation studies to show the performance of the proposed tracking controller are presented. We consider a mass-spring-damper system whose dynamics are described by

$$
\ddot{x}(t)+0.5 \dot{x}(t)+0.5 x(t)=u(t)+w(t),
$$

where $x(t), u(t)$ and $w(t)$ denote the displacement of the mass, the control input and the random disturbance, respectively. The system matrices of the discrete-time stochastic system (1) and (2) corresponding to (21) are given by

$$
\begin{aligned}
& A=\left[\begin{array}{cc}
1 & 0.01 \\
-5 \times 10^{-3} & 9.95 \times 10^{-1}
\end{array}\right] \\
& B=\left[\begin{array}{c}
4.99 \times 10^{-5} \\
0.01
\end{array}\right] \\
& G=\left[\begin{array}{c}
1.50 \times 10^{-4} \\
2.99 \times 10^{-2}
\end{array}\right], \quad C=[1,0]
\end{aligned}
$$

and $W=1$, where the time interval was set as $\Delta t=0.01[\mathrm{~s}]$. The weight matrices of the cost functional were set as $Q=\operatorname{diag}\{1000,0,0\}, R=1$. The risk-sensitive parameter was set as $\theta=1.6074$ which is in the risk-averse case. Further a controller having the risk parameter $\theta=0$ (riskneutral case), which is equivalent to an LQG controller, was also constructed for the sake of comparison.

The transient response of the controlled displacement $x\left(t_{k}\right)$ in the cases of $h=1$ and 20 are shown in Fig.1, where $\theta=1.6074$. The broken line indicates the reference signal $r\left(t_{k}\right)$ that is given by (3) with $\bar{r}=0.5$, where $t_{k}=k \Delta t$. The thin solid line indicates the transient response $x\left(t_{k}\right)$ controlled by the proposed controller with $h=1$. The thick solid line depicts the response of the displacement $x\left(t_{k}\right)$ controlled by the controller with $h=20$. Fig.2 shows the tracking errors in cases of $h=1$ and 20. It can be seen that the controller with the preview steps $h=20$ improved the amplitude of the tracking error, in comparison with the case of $h=1$.

Fig.3 shows the transient response of the displacement $x(t)$ in cases of $\theta=0$ (risk-neutral) and 1.64074 (risk-averse). The thick and thine solid lines depict the displacements in the cases that the 


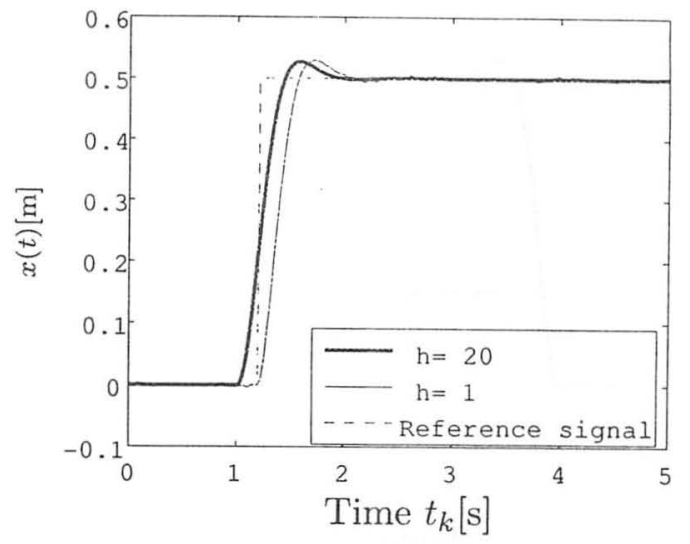

Fig. 1: Sample runs of the displacements of the mass $x(t)$ subject to the white Gaussian noise with $W=1$ in the cases of $h=1$ and 20 , where $t_{k}=$ $k \Delta t$.

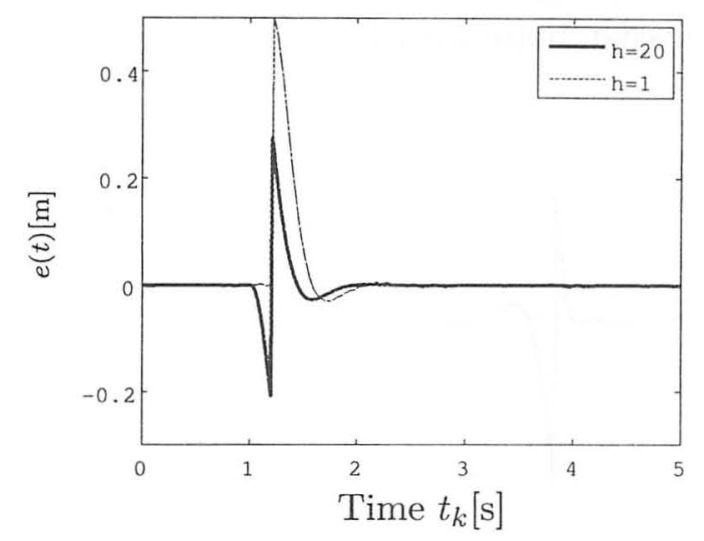

Fig. 2: Tracking errors in cases of $h=1$ and 20 with $\theta=1.6074$ and $W=1$.

controllers have been designed with $\theta=1.6074$ and 0 , respectively. The response of the output in the case of the controller designed with $\theta=1.6074$ shows that the peak value of the overshoot is less than that in the case of $(\theta=0)$. The tracking errors and the incremental controls are shown in Figs.4-6.

Figs.7-10 depict the transient responses of the displacements, tracking errors and incremental controls in the case that the controller designed with $\theta=1.6074$ and $W=1$ is used for the system subject to the white Gaussian noise with its covariance $W=100$. As seen in Fig.7, the response of the displacement controlled by the risk-sensitive control $(\theta=1.6074)$ is quite stable in the steady state. However the response by using the LQG control $(\theta=0)$ is fluctuated by the random disturbance.

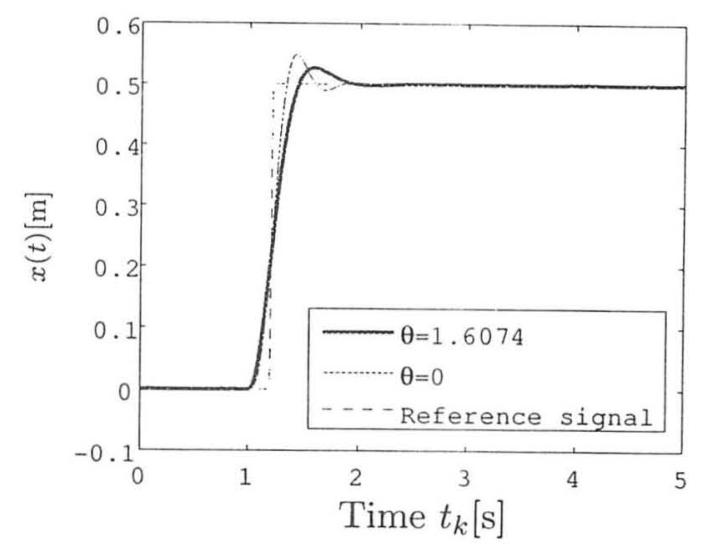

Fig. 3: Sample runs of the displacements of the mass $x(t)$ subject to the white Gaussian noise with $W=1$ in the cases of $\theta=0$ (risk-neutral) and 1.6074 (risk-averse), where $h=20$.

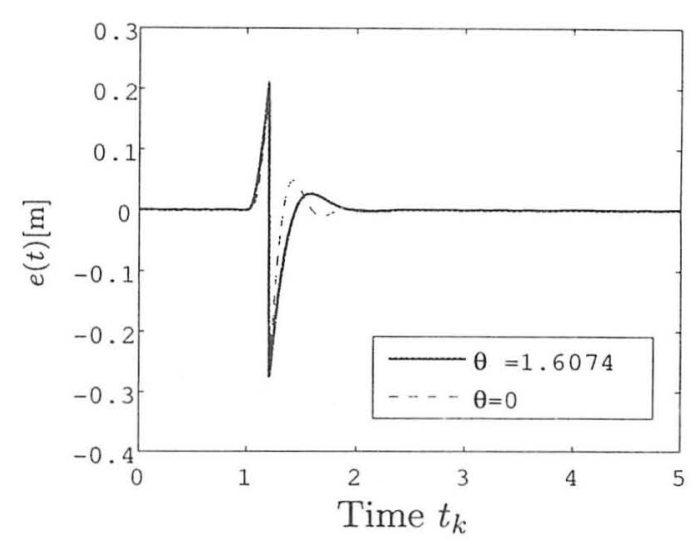

Fig. 4: Tracking errors $e(t)$ in the cases of $\theta=$ 0 and 1.6074 , where the system is subject to the white Gaussian noise with $W=1$.

\section{Conclusion}

This paper has presented a design method of risk-sensitive preview tracking control for discrete-time stochastic systems. The augmented system composed by the error system, incremental state system and the command generator is used for the design of the risk-sensitive control. This tracking control problem has been formulated as a risk-sensitive control problem for full-observed discrete-time stochastic linear systems. By performing the numerical simulations, it was shown that the proposed tracking controller has been more robust and stable for the strong random disturbance in the steady state than the LQG control. 


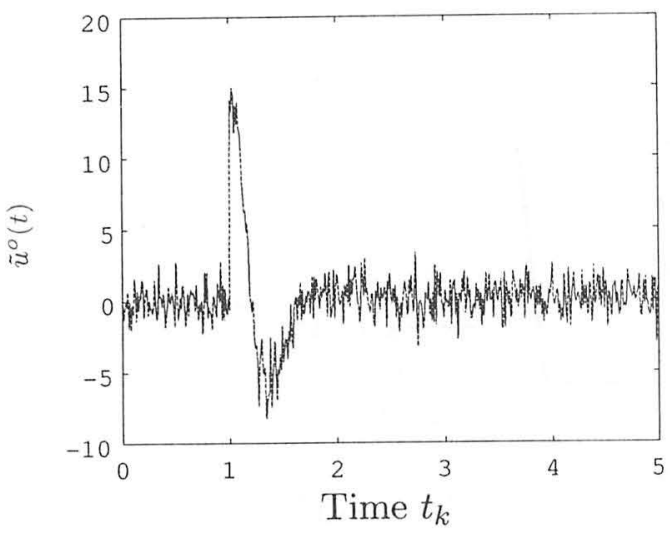

Fig. 5: Incremental control $\tilde{u}^{o}(t)(W=1, \theta=$ 1.6074).

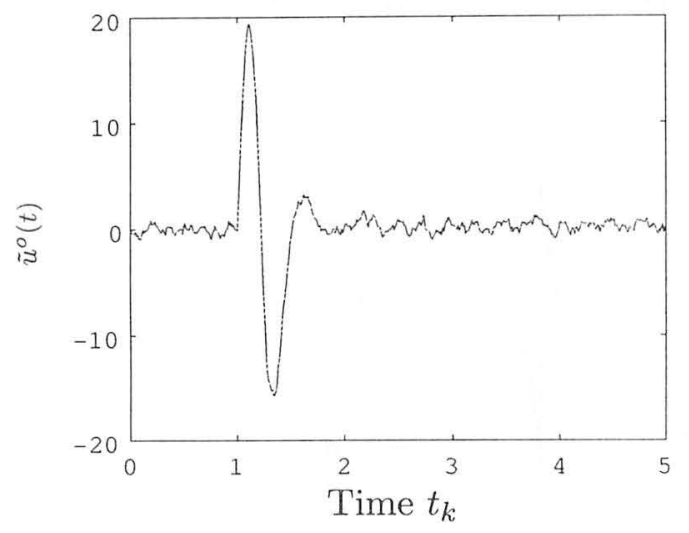

Fig. 6: Incremental control $\tilde{u}^{o}(t)(W=1, \theta=0)$.

\section{Acknowledgments}

The author wishes to thank Professor Akira Ohsumi, Kyoto Institute of Technology for his useful suggestions and advices. Part of this work was supported by the Ministry of Education, Culture, Sports, Science and Technology of Japan under the Grants-in-Aid for Scientific Research (C)-17560209 and (B)-16360046.

\section{References}

[1] M. Tomizuka, Optimal Continuous Finite Preview Problem, IEEE Trans. Automat. Contr., AC-20, No.3, pp.362-365, 1975.

[2] M. Tomizuka, On the Design of Digital Tracking COntrollers, Trans. ASME J. Dynamic Systems, Measurement, and Control, Vol.115, pp.412-418, 1994.

[3] T. Katayama, T. Ohki, T. Inoue and T. Kato, Design of an Optimal Controller for

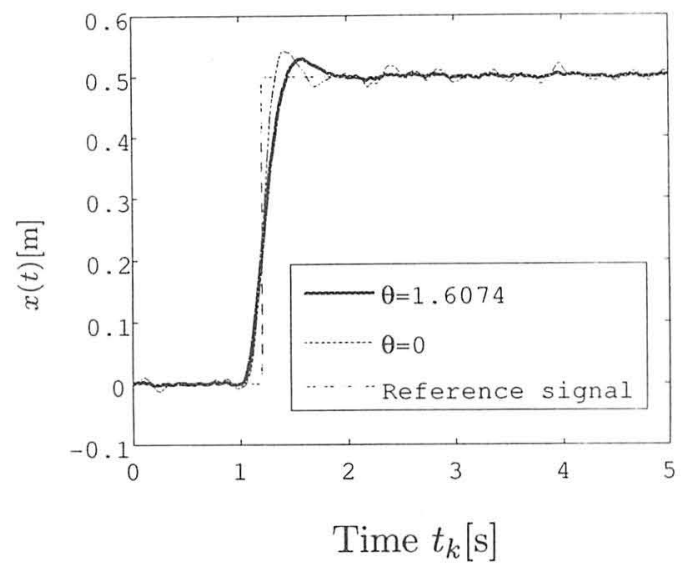

Fig. 7: Sample runs of the displacements $x(t)$ in the cases of $\theta=0$ and 1.6074, where $t=k \Delta t$, where the system in these cases are subject to the white Gaussian noise with $W=100$. However the controller is designed for the system subject to the white Gaussian noise with $W=1$.

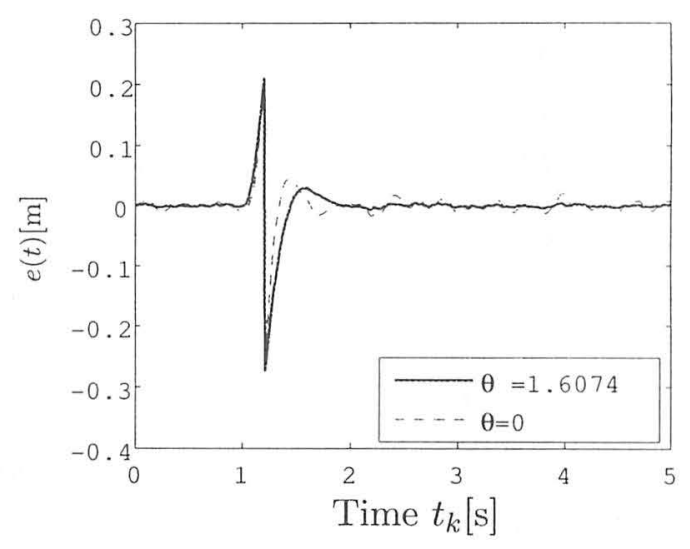

Fig. 8: Tracking errors $e(t)$ in the cases of $\theta=$ 0 and 1.6074, where the system is subject to the white Gaussian noise with $W=100$.

a Discrete-time System Subject to Previewable Demand, Int. J. Control, Vol.41, No.3, pp.677-699, 1985.

[4] A. Kojima and S. Ishijima, LQ Preview Synthesis: Optimal Control and Worst Case Analysis, IEEE Trans. Automat. Contr., AC44, No.2, pp.352-357, 1999.

[5] Y. Fujisaki and T. Narazaki, Optimal Preview Control Based on Quadratic Performace Index, Proc. 36th IEEE Conf. Decision and Control, pp.3830-3835, 1997.

[6] A. Cohen and U. Shaked, Linear Dsicretetime $H^{\infty}$-optimal Tracking with Preview, 


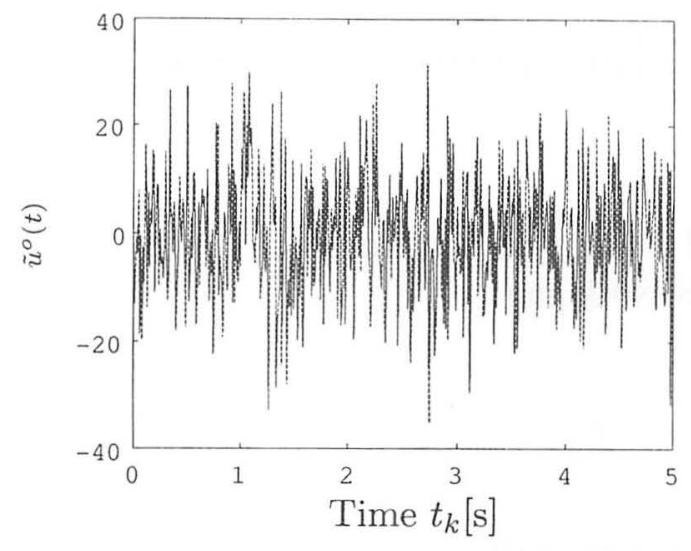

Fig. 9: Incremental control $\tilde{u}^{o}(t)$ designed for the system subject to the white Gaussian noise with $W=1$ and $\theta=1.6074$. The simulation was performed using the white Gaussian noise with $W=100$.

AC-42, No.2, pp.270-276, 1997.

[7] K. Takaba, Robust Preview Tracking Control for Polytopic Uncertain Systems, Proc. 37th IEEE Conf. Decision and Control, pp.17651770, 1998.

[8] L. Mianzo and H. Peng, A Unified Framework for LQ and $H_{\infty}$ Preview Control Algorithms, Proc. IEEE Conf. Decision and Control, pp.2816-2821, 1998.

[9] H.-S. Rho and Y. Park, Stochastic Optimal Preview Control of an Active Vehicle Suspension, J. Sound and Vibration, Vol.220, No.2, pp. 313-330, 1999.

[10] I. B. Collings, M. R. James and J. B. Moore, An Information-state Approache to Risk-sensitive Tracking Problems, J. Mathematical Systems, Estimation and Control, Vol.6, No.3, pp.1-24, 1996.

[11] D. H. Jacobson, Optimal Stochastic Linear Systems with Exponential Performance Criteria and Their Relation to Deterministic Differential Games, IEEE Trans. Automatic Control, AC-18, No.2, pp.124-131, 1973.

[12] J. L. Speyer, J. Deyst and D. H. Jacobson, Optimization of Stochastic Linear Systems with Additive Measurement and Process Noise Using Exponential Performance Criteria, IEEE Trans. Automat. Contr., AC-19, No.4, pp.358-366, 1974.

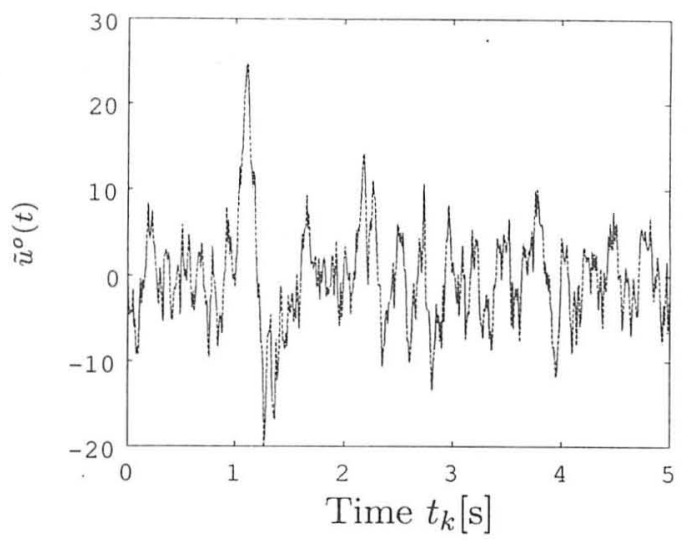

Fig. 10: Incremental control $\tilde{u}^{o}(t)$ designed for the system subject to the white Gaussian noise with $W=1$ and $\theta=0$ (LQG). The simulation was performed using the white Gaussian noise with $W=100$.

[13] A. Bensoussan and J. H. Van Schuppen, Optimal Control of Partially Observable Stochastic Systems with an Exponential-of-integral Performance Index, SIAM J. Control and $\mathrm{Op}$ timization, Vol.23, No.4, pp.599-613, 1985.

[14] A. Bensoussan, Stochastic Control of Partially Observable Systems, Cambridge University Press, United Kingdom, 1992.

[15] P. Whittle, A Risk-sensitive Maximum Principle: The Case of Imperfect State Observation, IEEE Trans. Automat. Contr., AC-36, No.7, pp.793-801, 1991.

[16] T. Runolfsson, The Equivalence Between Infinite-horizon Optimal Control of Stochastic Systems with Exponential-of-integral Performance Index and Stochastic Differential Games, IEEE Trans. Automat. Contr., AC39, No.8, pp.1551-1563, 1994.

[17] C.-H. Won and K. T. Gunaratne, Performance Study of LQG, MCV, and Risksensitive Control Methods for Satelite Structure Control, Proc. American Control Conference, pp.2481-2486, 2002.

[18] C.-H. Won, Satelite Structure Control with Parameter Robust Risk-sensitive Control Synthesis, Proc. American Control Conference, pp.3538-3542, 2004. 